\title{
$44 \quad$ INJURY PREVENTION COUNSELLING DURING WELL-CHILD VISITS IN THE USA: RATES AND DETERMINANTS
}

doi:10.1136/injuryprev-2012-040590d.44

${ }^{1} \mathrm{KF}$ Carlson, ${ }^{1} \mathrm{AG}$ Fernandez, ${ }^{2} \mathrm{AD}$ Ryan, ${ }^{3} \mathrm{ME}$ Betz. ${ }^{1}$ Portland Veterans Affairs Medical Center and Oregon Health and Science University, Portland, Oregon, USA; ${ }^{2}$ University of Minnesota School of Public Health, Minneapolis, Minnesota, USA; ${ }^{3}$ University of Colorado School of Medicine, Denver, Colorado, USA

Background Pediatric and adolescent injury prevention counselling (IPC) is recommended by USA medical organisations. Current rates and determinants of IPC in US child preventive care ('well-child') visits are unknown.

Aims/Objectives/Purpose We examined rates and determinants of IPC during well-child visits in US ambulatory care encounters.

Methods Data were from the 2009 National Ambulatory Medical Care Survey, a probability sample survey conducted annually by the US National Center for Health Statistics and designed to represent all US office-based physician visits. We conducted descriptive analyses on rates of IPC by patient characteristics and estimated the likelihood of IPC in reference to hypothesised determinants. OR and 95\% CI were calculated using multivariable logistic regression.

Results/Outcome There were approximately 57 million (95\% CI 47.2 to 66.8 ) well-child visits in US office-based physician clinics in 2009; 24\% involved IPC. Patient gender was not associated with IPC, but age, race/ethnicity, and region were. Rates of IPC decreased from $28 \%$ in visits with children aged $0-2$ years to $17 \%$ in those aged 13-18 years. Rates were lower for Hispanic (21\%) and Black (19\%) patients than for non-Hispanic Whites (25\%). In multivariable models, visits occurring in non-metropolitan regions were less likely to involve IPC (11\%) than visits in metropolitan (urban) regions (26\%; OR 0.4 ; $95 \%$ CI 0.2 to 0.7 ).

Significance/Contribution to the Field IPC can be effective in reducing injury among paediatric patients. Public health efforts should focus on increasing overall rates of IPC. Emphasis on children who are older, racial/ethnic minority, and seeking preventive care in rural regions may be needed. 